\title{
Artrite Crônica e Periodontite
}

\section{Chronic Arthritis and Periodontitis}

\author{
Flávia Silva Farah Ferreira Braga ${ }^{(1)}$, Letícia Algarves Miranda ${ }^{(2)}$, Vivian de Carvalho Miceli ${ }^{(3)}$, \\ Alessandra Áreas ${ }^{(4)}$, Carlos Marcelo Silva Figueredo ${ }^{(5)}$, Ricardo Guimarães Fischer ${ }^{(6)}$, Alessandra Fonseca \\ Graça da Silva Marques ${ }^{(7)}$, Luciene Lima Campos ${ }^{(8)}$, Flavio Roberto Sztajnbok ${ }^{(9)}$
}

\section{RESUMO}

Como parecem existir similaridades entre os mecanismos patogenéticos de doenças reumatológicas, como a artrite reumatóide e a artrite idiopática juvenil com a periodontite, alguns estudos têm sido publicados com o objetivo de levantar evidências de uma possível interrelação entre essas condições. A artrite reumatóide parece modular a resposta imune do hospedeiro, podendo aumentar a suscetibilidade à doença periodontal destrutiva em adultos. Recentemente, evidenciou-se que também pacientes com artrite idiopática juvenil possuíam maior suscetibilidade à doença periodontal destrutiva comparados a indivíduos saudáveis da mesma idade. No entanto, ainda se desconhecem os mecanismos que justificariam uma hipótese de associação entre essas condições crônicas inflamatórias. Sendo assim, o objetivo deste trabalho foi promover uma revisão da literatura sobre uma possível relação entre artrite crônica e periodontite.

Palavras-chave: artrite crônica, artrite idiopática juvenil, periodontite, artrite reumatóide.

\section{INTRODUÇÃO}

A artrite crônica e a periodontite apresentam similaridades em seus mecanismos patogênicos, o que tem despertado interesse na pesquisa sobre a associação entre essas condições $^{(1)}$. A artrite reumatóide (AR) pode funcionar como um modulador para a resposta imune no periodonto do hospedeiro, aumentando a suscetibilidade à doença periodontal destrutiva em adultos ${ }^{(2)}$. Recentemente, nosso grupo de pesquisa observou que pacientes com artrite idiopática juvenil (AIJ) possuíam maior freqüência de perda de inserção clínica periodontal na região interproximal, com-

\begin{abstract}
As similarities between pathogenetic mechanisms concerning rheumatic diseases, such as rheumatoid arthritis and juvenile idiopathic arthritis, with periodontitis may exhist, some studies have been published with the objective of showing evidences of a possible relationship between these conditions. Rheumatoid arthritis seems to modulate the host's immune mechanisms and may increase the susceptibility of adults to destructive periodontal disease. Recently evidences showed that also patients with juvenile idiopathic arthritis had an increased susceptibility to destructive periodontal disease compared to healthy individuals of the same age. Nevertheless the mechanisms of association of these chronic inflammatory conditions remain unclear. So, this study aims to review literature concerning a possible relationship between chronic arthritis and periodontitis.
\end{abstract}

Keywords: chronic arthritis, juvenile idiopathic arthritis, periodontitis, rheumatoid arthritis.

parados a indivíduos saudáveis da mesma idade ${ }^{(3)}$. Sendo assim, este trabalho tem como objetivo realizar uma revisão da literatura em relação a evidências sobre uma possível associação entre a AR, AIJ e a periodontite.

\section{PERIODONTITE}

O periodonto é definido como o conjunto de tecidos que circundam os dentes. Subdivide-se em periodonto de inserção (ligamento periodontal, cemento radicular e osso alveolar) e periodonto de proteção (gengiva) (Figura 1 ). A principal função do periodonto é inserir o dente no tecido

Recebido em 14/03/07. Aprovado, após revisão, em 23/05/07. Declaramos a inexistência de conflitos de interesse.

Estudo realizado no Setor de Pós-Graduação em Periodontia da Faculdade de Odontologia e no Setor de Reumatologia do Núcleo de Estudos da Saúde do Adolescente (Nesa) da Universidade do Estado do Rio de Janeiro (UERJ).

1. Mestre em Periodontia pela UERJ.

2. Professora Doutora de Periodontologia da Pontifícia Universidade Católica do Rio Grande do Sul

3. Mestre em Periodontia pela UERJ.

4. Doutora em Periodontia pela UERJ

5. Professor Adjunto Doutor de Periodontia da UERI

6. Professor Titular de Periodontia da UERJ

7. Médica Associada do Setor de Reumatologia do Nesa da UERJ.

8. Médica Associada do Setor de Reumatologia do Nesa da UERJ.

9. Responsável pelo Setor de Reumatologia do Nesa da UERJ. Professor Assistente do Departamento de Pediatria da Universidade Federal do Rio de Janeiro e da Unigranrio, Médico do Setor de Reumatologia Pediátrica do IPPMG-UFRJ.

Endereço para correspondência: Flavio R. Sztajnbok, Rua Álvaro Ramos, 405/804, bloco 1, CEP 22280-110, Rio de Janeiro, RJ, Brasil, e-mail: flavios@skydome.net ou flaviosztajnbok@hotmail.com 
ósseo dos maxilares e manter a integridade da superfície da mucosa mastigatória da cavidade oral. O grau de destruição que o periodonto apresenta num dado momento é medido por meio de uma sonda graduada em milímetros (sonda periodontal) (Figura 2). Com esse instrumento, podemos medir a profundidade do sulco gengival em seis faces por dente, bem como o nível de inserção. Além disso, sítios que apresentam inflamação no momento da sondagem sangram com o toque do instrumento, gerando um indicador de inflamação periodontal denominado sangramento à sondagem.

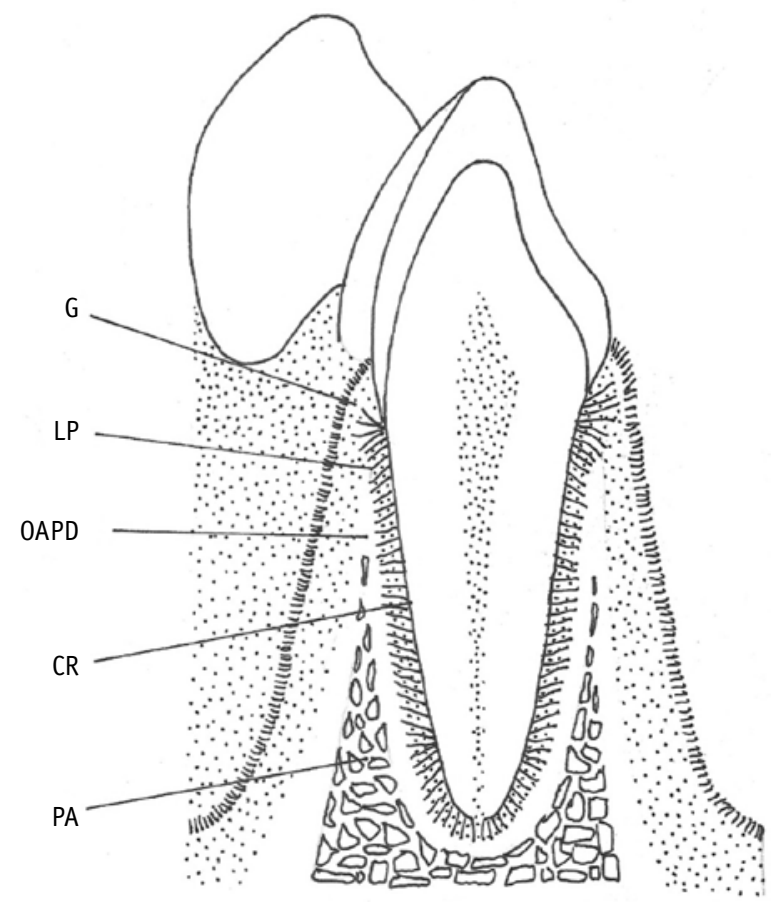

Figura 1 - Desenho esquemático do dente com seu periodonto: a gengiva $(G)$, o ligamento periodontal (LP), o cemento radicular (CR) e o osso alveolar: osso alveolar propriamente dito (OAPD) e o processo alveolar (PA).

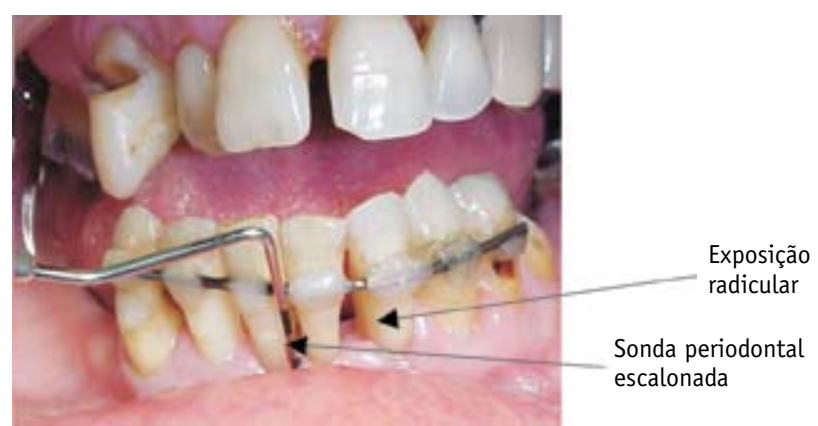

Figura 2 - Medição da profundidade de bolsa mediante a utilização de sonda periodontal calibrada em um paciente portador de periodontite crônica. Observe uma profundidade de aproximadamente $6 \mathrm{~mm}$ (marcação intercalada em preto na sonda de 3 em $3 \mathrm{~mm}$ totalizando $12 \mathrm{~mm}$ ) e extensa retração do tecido gengival expondo parcialmente as raízes dentárias.
O sulco gengival é o espaço entre a gengiva e o dente que se estende da margem gengival até o fundo do sulco sondável. Em condições normais, o sulco apresenta uma profundidade à sondagem inferior ou igual a 3 milímetros $(\mathrm{mm})$. Quando ocorre o aprofundamento do sulco gengival, isto é, quando a distância entre a margem gengival e o fundo da bolsa ultrapassa $3 \mathrm{~mm}$, diz-se que houve formação da bolsa periodontal. A bolsa periodontal verdadeira acompanha-se da perda dos tecidos de inserção periodontal. O nível de inserção periodontal é definido clinicamente como a distância entre a junção cementoesmalte (JCE) e o fundo da bolsa ou sulco. Uma vez que a gengiva está inserida no dente na altura da JCE, o nível de inserção clínica onde não ocorreu perda tecidual é zero. O sangramento à sondagem, o nível de placa bacteriana na superfície dental, a profundidade de bolsa à sondagem e as medidas do nível de inserção periodontal compõem os indicadores clínicos periodontais.

As principais condições inflamatórias que acometem o periodonto são gengivite e periodontite. A gengivite é uma inflamação apenas do periodonto de proteção e é clinicamente caracterizada por mudanças na coloração do tecido gengival (hiperemia) e presença de sangramento à sondagem e, normalmente, associa-se à presença de placa bacteriana no sulco gengival. Uma vez estabelecida a gengivite, se não houver interferência na formação continuada da placa bacteriana, pode desenvolver-se, em indivíduos suscetíveis, um quadro de periodontite.

A periodontite é uma inflamação crônica e destrutiva que leva à perda do tecido de suporte dos dentes e, eventualmente, à perda dentária e ao edentulismo. $\mathrm{O}$ ligamento periodontal e o tecido ósseo são destruídos por uma resposta imunológica à presença de bactérias no sulco gengival, especialmente as Gram-negativas, gerando inflamação. Essa destruição é, provavelmente, mediada por uma resposta alterada do hospedeiro, tornando-o suscetível ao desafio bacteriano. Ainda não está completamente esclarecido por que em alguns indivíduos a inflamação periodontal progride para periodontite e, em outros, restringe-se apenas à gengivite. No entanto, acredita-se que a progressão para periodontite possivelmente ocorra em virtude de uma combinação de eventos, incluindo o acúmulo de bactérias periodontopáticas, o aumento dos níveis de citocinas próinflamatórias, de enzimas proteolíticas e de prostaglandina $\mathrm{E}_{2}\left(\mathrm{PGE}_{2}\right)$, somados a uma redução dos níveis de antagonistas de citocinas e inibidores de proteases ${ }^{(4)}$. Na literatura, alguns fatores, como o fumo, têm sido considerados modificadores da resposta imunológica do hospedeiro e 
podem ser determinantes na variação à suscetibilidade à periodontite $^{(5)}$.

Assim, a periodontite pode ser considerada uma inflamação que vai além da gengiva, alcançando o tecido ósseo subjacente, o ligamento periodontal e o cemento radicular, levando à formação da bolsa periodontal. Clinicamente, além da presença da bolsa periodontal, podemos observar a perda da inserção periodontal e, radiologicamente, a de tecido ósseo.

Estudos têm evidenciado uma possível associação entre a periodontite e algumas condições reumatológicas, onde está presente a artrite crônica ${ }^{(2,3,6,7)}$.

\section{HIPÓTESES DE ASSOCIAÇÃO ENTRE A AR- TRITE CRÔNICA E A PERIODONTITE}

Há estudos sugerindo que condições reumatológicas, como a AR e a AIJ, também possam ser modificadoras do processo saúde-doença periodontal, aumentando a suscetibilidade à doença periodontal destrutiva, tanto em adultos ${ }^{(2,6)}$ como em crianças e adolescentes ${ }^{(3,7)}$. No entanto, esses resultados não são corroborados por outros estudos $^{(8)}$. A Tabela 1 descreve os principais trabalhos interrelacionando a AR e a AIJ com condições orais.

Algumas hipóteses têm sido sugeridas para justificar uma possível associação entre a AR e a periodontite. Tais hipóteses serviriam também, com plausibilidade biológica, para uma inter-relação entre a AIJ e a periodontite, uma vez que, em todas essas condições, evidencia-se uma importante desregulação do sistema imune, possivelmente associada a ou influenciada por fatores genéticos e ambientais. A inter-relação entre a artrite crônica e a periodontite parece ter um caráter bidirecional.

Uma das teorias que tenta explicar como a periodontite seria uma condição de risco à AR baseia-se na exposição crônica ao lipopolissacarídeo que ocorre nas doenças periodontais. Segundo esse conceito, o lipopolissacarídeo de bactérias periodontopáticas serviria como uma fonte de superantígenos ao hospedeiro, podendo iniciar a cascata imunológica observada na $\mathrm{AR}^{(9)}$. Por outro lado, a desregulação imunológica observada na AR, gerando o aumento de citocinas como a interleucina 1 (IL-1), o fator de necrose tumoral (TNF- $\alpha$ ) e a IL-6, local e sistemicamente, faria com que pacientes com artrite reumatóide, na presença de patógenos periodontais e um meio ambiente propício, desenvolvessem maior suscetibilidade à periodontite ${ }^{(6)}$. Além disso, a hiperatividade neutrofilica tem sido evidenciada em certas doenças crônicas e parece justificar possíveis inter-relações entre algumas condições inflamatórias. Os neutrófilos são as células mais importantes nas articulações de pacientes com AR ativa e parecem desempenhar uma importante função na periodontite. Evidenciou-se hiperatividade de neutrófilos tanto na periodontite quanto na artrite. Desta forma, talvez uma doença possa funcionar como estímulo pré-ativador para neutrófilos periféricos, fazendo que essas

TABELA 1

ESTUDOS INTER-RELACIONANDO A AIJ E A AR COM CONDIÇÕES ORAIS

\begin{tabular}{|c|c|}
\hline Autores & Principais achados \\
\hline Sjöström et al. ${ }^{(8)}$ & $\begin{array}{l}\text { Estudo transversal que mostrou haver uma tendência para melhores indicadores periodontais em pacientes com AR ( } \mathrm{n}=204) \text {, sem } \\
\text { significância estatística, com exceção dos níveis de placa, significativamente menores no grupo AR }\end{array}$ \\
\hline Kasser et al. ${ }^{(16)}$ & $\begin{array}{l}\text { Estudo transversal em que pacientes com AR }(n=50) \text { apresentaram níveis de placa semelhantes, maior número de dentes ausentes, } \\
\text { mais sangramento gengival, mais bolsas profundas e maior perda de inserção que controles. Não se verificou correlação entre medidas } \\
\text { de AR e variáveis periodontais. Controlado para higiene oral, fumo, sexo e idade }\end{array}$ \\
\hline Mercado et al. ${ }^{(2)}$ & $\begin{array}{l}\text { Estudo transversal que avaliou pacientes referidos para tratamento periodontal }(n=809) \text {. A prevalência de AR auto-reportada foi } \\
\text { maior do que em pacientes sem periodontite }(n=603)\end{array}$ \\
\hline Mercado et al. ${ }^{(6)}$ & $\begin{array}{l}\text { Estudo transversal que mostrou que pacientes com AR }(n=65) \text { apresentaram mais dentes ausentes e maior porcentagem de bolsas } \\
\text { profundas }(\geq 6 \mathrm{~mm}) \text { que controles, embora apresentassem níveis de placa e sangramento similares. Constataram-se associações sig- } \\
\text { nificativas entre perda óssea alveolar e os seguintes parâmetros de AR: número de articulações edemaciadas, escores de HAQ, níveis } \\
\text { de PCR e VHS. Pacientes com AR e periodontite moderada a severa apresentaram maior número de articulações edemaciadas }\end{array}$ \\
\hline Miranda et al. ${ }^{(3)}$ & $\begin{array}{l}\text { Estudo transversal que mostrou que pacientes com AIJ }(n=32) \text { possuíam maior freqüência de perda de inserção interproximal com- } \\
\text { parados a controles saudáveis }\end{array}$ \\
\hline Miranda et al. ${ }^{(17)}$ & $\begin{array}{l}\text { Estudo transversal que mostrou que pacientes com AIJ }(n=38) \text { possuíam níveis sorológicos elevados de IL-18, especialmente na } \\
\text { presença de perda de inserção periodontal }\end{array}$ \\
\hline Havemose-Poulsen et al. ${ }^{(15)}$ & Estudo transversal que mostrou que pacientes com AR $(n=23)$ possuíam mais bolsas profundas que indivíduos controles \\
\hline Miranda et al. ${ }^{(7)}$ & $\begin{array}{l}\text { Estudo longitudinal que mostrou que pacientes portadores de AIJ }(n=18) \text { que apresentaram melhora clínica e laboratorial após } 2 \text { anos } \\
\text { de tratamento não mostravam diferença estatisticamente significativa em relação a controles no tocante à inflamação periodontal } \\
\text { clínica e/ou laboratorial }\end{array}$ \\
\hline
\end{tabular}

DMARD = drogas anti-reumáticas modificadoras de doença; $\mathrm{PCR}=$ proteína $\mathrm{C}$ reativa; $\mathrm{HAQ}=$ questionário de avaliação de saúde; $\mathrm{VHS}=$ velocidade de hemossedimentação. 
células ajam de forma mais agressiva, quando recrutadas para atuar em outra doença ${ }^{(10-12)}$.

O tratamento inicial clássico para pacientes com artrite crônica inclui a utilização de antiinflamatórios não esteróides (AINEs), que têm sido estudados como adjuntos no tratamento periodontal. Um estudo retrospectivo mostrou menor perda óssea alveolar em pacientes portadores de AR que faziam uso de AINEs, comparados a um grupo de indivíduos saudáveis que não usava medicação ${ }^{(13)}$. Por outro lado, outros autores ${ }^{(14)}$ observaram que pacientes que faziam uso crônico desse tipo de medicamento não apresentavam diferenças no índice de placa, índice gengival, profundidade de bolsa, perda de inserção periodontal e perda óssea comparados a indivíduos não medicados.

\section{DISCUSSÃO}

O conhecimento de que o sistema imune participa ativamente da patogênese da AR, AIJ e periodontite tem estimulado a realização de vários estudos para avaliar o status periodontal em pacientes com AR e AIJ. Na AR, AIJ e periodontite, a presença de atividade clínica mostra, em nível sistêmico e local, um nível aumentado de citocinas pró-inflamatórias, além de baixos níveis de inibidores de metaloproteinases, altos níveis de metaloproteinases de matriz e prostaglandina $\mathrm{E}_{2}$ secretados por macrófagos, fibroblastos e outras células inflamatórias ${ }^{(4,10-12,15)}$. Alguns estudos demonstram piores condições periodontais em adultos e jovens, além de maior freqüência de perda dentária em pacientes com AR e AIJ, comparados a controles saudáveis ${ }^{(2,3,6,9,15,16)}$. Entretanto, outros estudos apontam uma evidência de condições similares ou até mesmo tendência para melhores indicadores periodontais

\section{REFERÊNCIAS}

1. Petty RE, Southwood TR, Manners P, et al.: International League of Associations for Rheumatology classification of juvenile idiopathic arthritis. 2nd revision. Edmonton, 2001. J Rheumatol 31(2): 390-2, 2004.

2. Mercado F, Marshal RI, Klestov AC, Bartold PM: Is there a relationship between rheumatoid arthritis and periodontal disease? J Clin Periodontol 27(4): 267-72, 2000.

3. Miranda LA, Fischer RG, Sztajnbok FR, Figueredo CMS, Gustafsson A: Periodontal conditions in patients with juvenile idiopathic arthritis. J Clin Periodontol 30(11): 969-74, 2003.

4. Page RC: Advances in the pathogenesis of periodontitis: summary of developments, clinical implications and future directions. Periodontol 2000 14:216-48, 1997. em pacientes com $\mathrm{AR}^{(8)}$. Diferenças nos critérios de doença e nos métodos para avaliar o status da doença periodontal formam o principal problema na interpretação desta literatura. Além disso, muito embora o uso prolongado de AINEs possa não alterar os índices clínicos periodontais ${ }^{(14)}$, há evidência de que essas drogas, utilizadas durante um longo período de tempo, afetam a progressão da perda óssea periodontal ${ }^{(13)}$. Desta forma, a falha em demonstrar uma inter-relação entre a artrite crônica e a periodontite talvez possa ser atribuída ao uso crônico de AINEs pelos pacientes com AR e AIJ. Além disso, os estudos disponíveis sobre o status periodontal em portadores de alterações reumatológicas são transversais, tornando difícil avaliar pontos importantes como as causas de perda dentária. Recentemente, um estudo longitudinal que acompanhou pacientes com AIJ por 2 anos mostrou que, após controle clínico e laboratorial com tratamento adequado (que incluiu corticosteróide, metotrexato e AINEs), houve significativa diminuição nos níveis de IL-1 $\beta$ no fluido crevicular cervical, mas não houve diferença estatisticamente significativa, quando comparados ao grupo controle, em relação à inflamação periodontal clínica e laboratorial. Isto poderia indicar que, em relação ao binômio AIJ-periodontite, a doença controlada e inativa não mais influenciaria o status periodontal, ratificando a existência de uma inter-relação entre ambas as condições ${ }^{(7)}$.

Assim, se a AIJ se associa ou não à progressão de outras condições inflamatórias como a periodontite, ainda não está completamente esclarecido. Entretanto, a artrite e a periodontite parecem apresentar semelhanças em seus mecanismos patogenéticos. Novos trabalhos prospectivos são necessários para avaliar a associação entre essas inflamações.

5. Kinane DF, Chestnutt IG: Smoking and periodontal disease. Crit Rev Oral Biol Med 11(3): 356-65, 2000.

6. Mercado FB, Marshal RI, Klestov AC, Bartold PM: Relationship between rheumatoid arthritis and periodontitis. J Periodontol 72(6): 779-87, 2001.

7. Miranda LA, Braga F, Fischer RG, Sztajnbok FR, Figueredo CMS, Gustafsson A: Changes in periodontal and rheumatological conditions after 2 years in patients with juvenile idiopathic arthritis. J Periodontol 77: 1695-700, 2006.

8. Sjostrom L, Laurell L, Hugoson A, Hakansson JP: Periodontal conditions in adults with rheumatoid arthritis. Community Dent Oral Epidemiol 17(5): 234-6, 1989.

9. Mercado FB, Marshall RI, Bartold PM: Inter-relationships between rheumatoid arthritis and periodontal disease. J Clin Periodontol 30(9): 761-72, 2003. 
10. Pillinger $\mathrm{MH}$, Abramson SB: The neutrophil in rheumatoid arthritis. Rheum Dis Clin North Am 21(3): 691-714, 1995.

11. Figueredo CMS: Hyperreactive neutrophils in periodontitis (Thesis). In: Periodontology. Stockholm: Karolinska Institutet, 1999.

12. Kitsis E, Weissmann G: The role of the neutrophil in rheumatoid arthritis. Clin Orthop Relat Res 265: 63-72, 1991.

13. Feldman RS: Non-steroidal anti-inflammatory drugs in the reduction of human alveolar bone loss. J Clin Periodontol 10(2): $131-6,1983$

14. Heasman PA, Seymour RA: An association between long-term non-steroidal anti-inflammatory drug therapy and the severity of periodontal disease. J Clin Periodontol 17(9): 654-8, 1990.
15. Havemose-Poulsen A, Westergaard J, Stoltze K, et al. Periodontal and hematological characteristics associated with aggressive periodontitis, juvenile idiopathic arthritis and rheumatoid arthritis. J Periodontol 77(2): 280-8, 2006.

16. Kasser UR, Gleissner C, Dehne F, Michel A, Willerhausen BZ, Bolten W: Risk for periodontal disease in patients with longstanding rheumatoid arthritis. Arthritis Rheum 40(12): 2248-51, 1997.

17. Miranda LA, Fischer RG, Sztajnbok FR, Johansson A, Figueredo CMS, Gustafsson A: Increased interleukin-18 in patients with juvenile idiopathic arthritis and early attachment loss. J Periodontol 76(1): 75-82, 2005. 